\title{
Circular RNA expression profiles in neonatal rats following hypoxic-ischemic brain damage
}

\author{
LI JIANG ${ }^{1}$, HUIJUAN LI ${ }^{1}$, ZHONGMIN FAN $^{2}$, RUIBIN ZHAO ${ }^{3}$ and ZHENGKUN XIA ${ }^{4}$ \\ ${ }^{1}$ Department of Pediatrics, Zhongda Hospital, Southeast University, Nanjing, Jiangsu 210097; \\ ${ }^{2}$ Department of Pediatrics, The BenQ Hospital, Nanjing Medical University, Nanjing, Jiangsu 210019; \\ ${ }^{3}$ Department of Pediatrics, Affiliated Hospital of Yangzhou University, Yangzhou, Jiangsu 225000; \\ ${ }^{4}$ Department of Pediatrics, Jinling Hospital, Nanjing University, Nanjing, Jiangsu 210093, P.R. China
}

Received September 12, 2018; Accepted February 13, 2019

DOI: $10.3892 / \mathrm{ijmm} .2019 .4111$

\begin{abstract}
Circular RNAs (circRNAs) have been studied in a number of diseases. However, the roles of circRNAs in hypoxic-ischemic brain damage (HIBD) remains unknown. In the present study, high throughput sequencing was used to profile altered circRNAs in HIBD rats. A total of 66 circRNAs were identified to be differentially expressed (fold-change $>2$ and P-value $<0.05$ ) in HIBD rats compared with the control group, including 20 upregulated and 46 downregulated circRNAs. Gene ontology and Kyoto Encyclopedia of Genes and Genomes pathway analysis indicated that numerous mRNAs transcribed from the host genes of altered circRNAs were involved in brain damage and neural regeneration. The interaction of circRNA/microRNA was predicted based on TargetScan and miRanda. The results of this study demonstrated an altered circRNA expression pattern in HIBD rats and suggests important roles in HIBD physiological and pathological processes. These findings suggest a novel focus for future studies investigating the molecular mechanism underlying HIBD and possibilities for the treatment of HIBD through modulating circRNAs.
\end{abstract}

\section{Introduction}

Hypoxic ischemic encephalopathy (HIE) can cause neonatal morbidity and mortality (1). Advances in obstetrical and neonatal care has improved the survival rate of HIE resulting

Correspondence to: Dr Ruibin Zhao, Department of Pediatrics, Affiliated Hospital of Yangzhou University, 368 Hanjiang Middle Road, Yangzhou, Jiangsu 225000, P.R. China

E-mail: tczhaoruibin@163.com

Dr Zhengkun Xia, Department of Pediatrics, Jinling Hospital, Nanjing University, 22 Hankou Road, Nanjing, Jiangsu 210093, P.R. China

E-mail:njxzk@126.com

Key words: circular RNA, hypoxic-ischemic brain damage, high throughput sequencing, brain injury in an increase in the number of infants at risk of permanent neurological deficits (1). Therefore, HIE is still considered a threat to the quality of a child's health and life and further research is required to develop effective neurotherapeutic interventions for HIE.

Circular RNAs (CircRNAs) are a unique type of endogenous non coding RNAs that are formed by back-splicing events via protein coding exons $(2,3)$. They are characterized by conservation, stability, abundance and tissue/developmental stage-specific expression (4,5). Previously, it has been reported that circRNAs have multiple biological functions, which are involved in promoting rolling circle translation, controlling the transcription of parent genes, assisting the formation of alternatively spliced mRNA and acting as microRNA (miRNA/miR) sponges (6-10). Altered levels of specific circRNAs are reported to be associated with numerous human diseases, including cancer, ischemia, stroke, neurodegenerative diseases, heart disease and cartilage degradation $(11,12)$. Recent studies $(4,13)$ performed in adult rats demonstrate that cerebral circRNA expression profiles are significantly altered following stroke and contribute to the stabilization of mRNA expression (14). Few studies reported to date have evaluated circRNA alterations in a hypoxic ischemic (HI) injured brain.

To the best of the authors' knowledge, methods used for the identification of novel disease-associated circRNAs include microarray, reverse transcription-quantitative polymerase chain reaction (RT-qPCR), RNA-seq and capture sequencing (15). To determine whether hypoxic-ischemic brain damage (HIBD) influences the expression of circRNAs to identify novel targets for further studies, alteration of circRNA expression profiles in HIBD rat was investigated in this study using RNA-Seq. The function of circRNA may be associated with the known function of the host genes and therefore gene ontology (GO) and Kyoto Encyclopedia of Genes and Genomes (KEGG) pathway analysis were performed to predict potential functions of circRNAs in HIBD. Furthermore, miRNAs regulated by differentially expressed circRNAs were selected for further investigation using RT-qPCR. Using a combination of RNA-seq results, bioinformatics analysis and subsequent RT-qPCR, potential roles of these dysregulated circRNAs in pathophysiological processes in HIBD are proposed. 


\section{Materials and methods}

The study included 4 procedural modules, including library preparation, high throughput sequencing, bioinformatics analysis and RT-qPCR validation (Fig. 1).

Animals. Sprague-Dawley rats, (age 10 days after birth; $18-20 \mathrm{~g}$, without sex selection) were obtained from the Medical Animal Center of Nanjing Medical University (Nanjing, China). All rats were housed for $\geq 7$ days prior to the study in a temperature $\left(22-25^{\circ} \mathrm{C}\right)$ and humidity-controlled $(50 \%)$ animal facility under a $12 \mathrm{~h} \mathrm{light/dark} \mathrm{cycle.} \mathrm{Animals} \mathrm{had} \mathrm{free} \mathrm{access} \mathrm{to} \mathrm{food} \mathrm{and}$ water; however, food was withheld overnight prior to surgery. All animal procedures were approved by the Zhongda Hospital Committee on Animal Research and all the experiments were carried out in accordance with the approved guidelines.

Preparation of HIBD rats. HIBD was produced in rats according to previously described methods (16). Briefly, following anesthetization using ether, rats were subjected to ischemia, with the right common carotid artery permanently double ligated and sliced from the middle. Following a recovery time of $1 \mathrm{~h}$, the animals were exposed to hypoxia $\left(8 \% \mathrm{O}_{2}, 92 \% \mathrm{~N}_{2}\right)$ for $2.5 \mathrm{~h}$ and then returned to their normal living environment. Sham control rats were subjected to isolation and tying ligatures around vessels without occlusion and subsequent ischemia. A total of $24 \mathrm{~h}$ following $\mathrm{HI}$ insult, the rats were sacrificed using sodium pentobarbital intraperitoneally $(40 \mathrm{mg} / \mathrm{kg})$ and their ipsilateral hemispheres, including the cortex and hippocampus, were harvested. The tissues were stored in liquid nitrogen $\left(-196^{\circ} \mathrm{C}\right)$ until the following experiment was carried out.

RNA isolation and quality control. Total RNA was extracted from the hippocampus using TRIzol Reagent (Invitrogen; Thermo Fisher Scientific, Inc., Waltham, MA, USA) according to the manufacturer's protocol. The concentration and quality of RNA was assessed using NanoDrop ND-1000 spectrophotometry (NanoDrop, Wilmington, DE, USA) and RNA integrity was determined using $1 \%$ denaturing agarose gel electrophoresis.

Library preparation and RNA-seq. RNA-seq analysis was performed at the Decode Genomics Biotechnology Co., Ltd. (Nanjing, China). The sequencing library was prepared following the removal of ribosomal RNA, according to Illumina ${ }^{\circledast}$ TruSeq ${ }^{\circledR}$ RNA Sample Preparation Guide (Illumina, Inc., San Diego, CA, USA). Ligation of the indexed adaptor was performed following the synthesis of double strand cDNA. Following size selection using Agencourt AMPure XP (Beckman Coulter, Inc., Brea, CA, USA), the libraries were quantified and quality assessed using a Qubit ${ }^{\circledR} 2.0$ Fluorometer with the Qubit ${ }^{\circledR}$ HSDNA BR assay kit (Invitrogen ${ }^{\mathrm{TM}}$; Thermo Fisher Scientific, Inc.) and Agilent bioanalyzer (Agilent Technologies, Inc., Santa Clara, CA, USA), respectively. Sequencing was performed using an Illumina HiSeq X-10 (Illumina, Inc.) with 2x150 bp pair-end technology.

Bioinformatic analyses. Quality control of the sequencing was performed using FastQC version 0.11.2 (http://www. bioinformatics.babraham.ac.uk/projects/fastqc/). Raw data of poor quality ( $>50 \%$ bases having a low-quality threshold $<20$ ) and adapter sequences were removed using SOAPnuke, to improve results. The clean data was mapped to rat genomes, provided by Illumina iGenomes (downloaded from http:// cole-trapnell-lab.github.io/cufflinks/cuffdiff/index.html) with Tophat2 (version 2.0.7) calling Bowtie2 (version 2.1.0) using default settings. The read numbers mapped to each circRNA were counted. RPM Reads Per Million mapped reads, including TopHat mapping and TopHat-Fusion mapping, were used to calculate the expression level of individual circRNA. Differential expression between circRNAs was assessed using DEGseq algorithm (17). The statistical criteria for selecting aberrant-expressed circRNA was defined using a $q$-value [the false discovery rate adjusted P-value $<0.05$ with a fold-change $>2.0$ or $<0.5(18,19)]$. CircRNA annotation was based on the Gencode rat genome (v10) (20). The circRNA donator/acceptor site was intersected to annotate gene regions, including coding RNA, non-coding RNA, intron, antisense and intergenic regions, with the BEDTools suite (v2.16.2) (21). The number of reads aligning to the circRNA-specific head-to-tail junctions was used as a measurement of circRNAs expression.

GO analysis provides a controlled vocabulary to describe genes and gene product attributes in any organism (http://www. geneontology.org). This ontology includes three domains: Biological processes, cellular components and molecular functions. Fisher's exact test was used to detect overlapping data between the differentially expressed list and GO annotation list, except for that expected by chance. Pathway analysis was used to map genes to the KEGG pathways.

To investigate the regulation between circRNAs and mRNAs in the neonatal rats following HIBD, interaction networks were studied among the 6 differentially expressed circRNAs, whose host gene was located on the exon and was identified along with their corresponding target miRNAs. CircRNAs-targeted miRNAs were predicted using miRNA target prediction software based on TargetScan (http://www. targetscan.org/vert_71/) and miRanda (http://www.microrna. org/microrna/home.do). The co-expression network was illustrated using Cytoscape (v2.8.1, https://cytoscape.org/). The associated analyses and drawing were performed by Decode Genomics Biotechnology Co., Ltd. (Nanjing, China).

$R T$ - $q P C R$. Total RNA was extracted from hippocampus samples using TRIzol reagent (Invitrogen; Thermo Fisher Scientific, Inc.) and converted to cDNA using PrimeScript RT-PCR kit (cat. no. RR037A; Takara Bio, Inc., Otsu, Japan), according to the manufacturer's protocol. In brief, the reaction system was incubated at $25^{\circ} \mathrm{C}$ for $10 \mathrm{~min}$, and then at $42^{\circ} \mathrm{C}$ for $60 \mathrm{~min}$. PCR was performed in a total reaction volume of $25 \mu \mathrm{l}$, containing $12.5 \mu \mathrm{l}$ SYBR Premix Ex Taq (2X), $2 \mu 1 \mathrm{cDNA}, 1 \mu \mathrm{l}$ forward primer $(10 \mu \mathrm{M}), 1 \mu \mathrm{l}$ reverse primer $(10 \mu \mathrm{M}), 0.5 \mu \mathrm{l}$ ROX Reference Dye II (50X) and $8 \mu$ l double-distilled water. The amplification procedures were as follows: $10 \mathrm{~min}$ at $95^{\circ} \mathrm{C}$ to initiate denaturation; 40 cycles of $5 \mathrm{sec}$ at $95^{\circ} \mathrm{C}, 30 \mathrm{sec}$ at $63^{\circ} \mathrm{C}$ and $30 \mathrm{sec}$ at $72^{\circ} \mathrm{C}$; and a final extension of $5 \mathrm{~min}$ at $72^{\circ} \mathrm{C}$. Amplification efficiency was evaluated using standard curve analysis. All samples were normalized using the $2^{-\Delta \Delta C q}$ method against GAPDH and the experiment was repeated in triplicate (22). The primers of the circRNAs and GAPDH are listed in Table I. 
Table I. DNA sequence of reverse transcription-quantitative polymerase chain reaction primers used for analysis of circular RNA and mRNA level.

Name

DNA sequence

Product size (bp)

\section{GAPDH (Rat)}

chr11: 34065887134070810

chr10: 13931236113935484

chr1: 2008990661201028171

chr13: $31092209 \mid 31209056$

\section{F: 5'-GCTCTCTGCTCCTCCCTGTTCTA-3'}

R: 5'-TGGTAACCAGGCGTCCGATA-3'

F: 5'-CCTCTGCCTTGTTTTGCTGT-3'

R: 5'-GTTGGGGAGGAAAGGGTTTA-3'

F: 5'-TTTTCCACGATGAACAACC -3'

R: 5'-CAGCAGGTGGCAGCTGTAT-3'

F: 5'-ATTACCCATGCCCAGATGTT-3'

R: 5'-TGCAGTTTTCAAATGGGTCA-3'

F: 5'-CTAAACAGGGGCTTCTCAGC-3'

R: 5'-GTCATTGTTCTAAGCATCAGTGG-3'
124

146

88

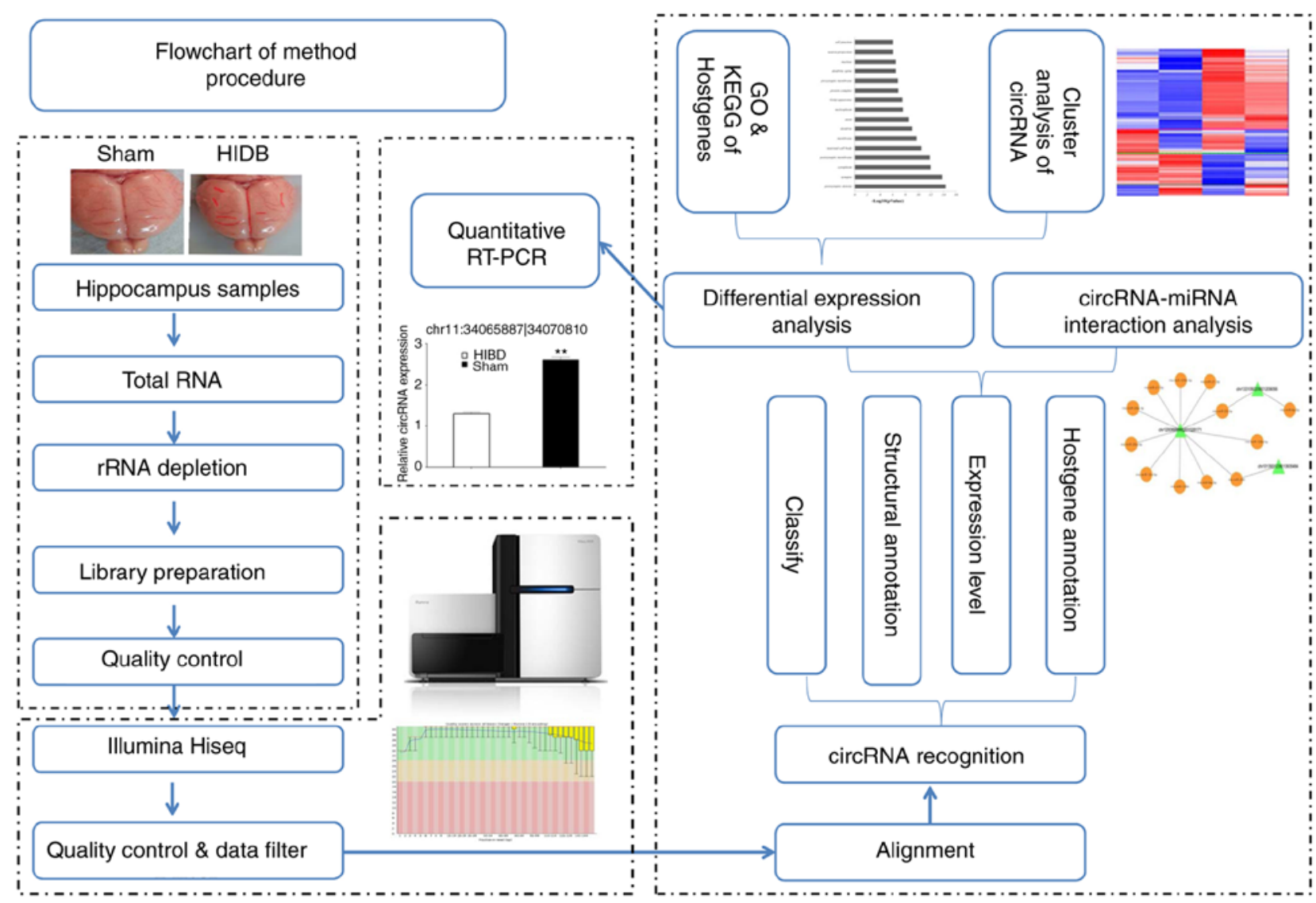

Figure 1. The flowchart of the experimental procedures. The scheme includes four steps. First, sample collection, RNA extraction and library preparation. Then, sequencing with Illumina Hiseq X10 and next, differentially expressed circRNAs and host genes in neonatal brains following hypoxic-ischemic brain injury compared with the sham control brains were identified. Subsequently, bioinformatics analysis, including GO and KEGG pathway enrichment were network performed for analyzing the correlation between circRNAs and miRNAs. Finally, RT-qPCR was utilized to validate the sequencing results. Circ, circular; miRNA, microRNA; RT-qPCR, reverse transcription-quantitative polymerase chain reaction; KEGG, Kyoto encyclopedia of genes and genomes; GO, gene ontology; HIBD, hypoxic-ischemic brain damage; rRNA, ribosomal.

Statistical analysis. Data analyses were performed using GraphPad Prism 5.0 (GraphPad, Inc., La Jolla, CA, USA), Cluster 3.0 (Human Genome Center, University of Tokyo, Tokyo, Japan) and Cytoscape 2.8.1 (https://cytoscape. org/). Data are presented as the with mean \pm standard error of the mean and three repeats were performed for each experiment. Statistical differences were determined using
Students t-test and one-way analysis of variance. Multiple comparisons between the groups were performed using the Student-Newman-Kuels method. K-means clustering algorithms were performed to classify the samples according to the selected differently expressed genes. All statistical tests were performed as two-sided tests and $\mathrm{P}<0.05$ was considered to indicate a statistically significant difference. 


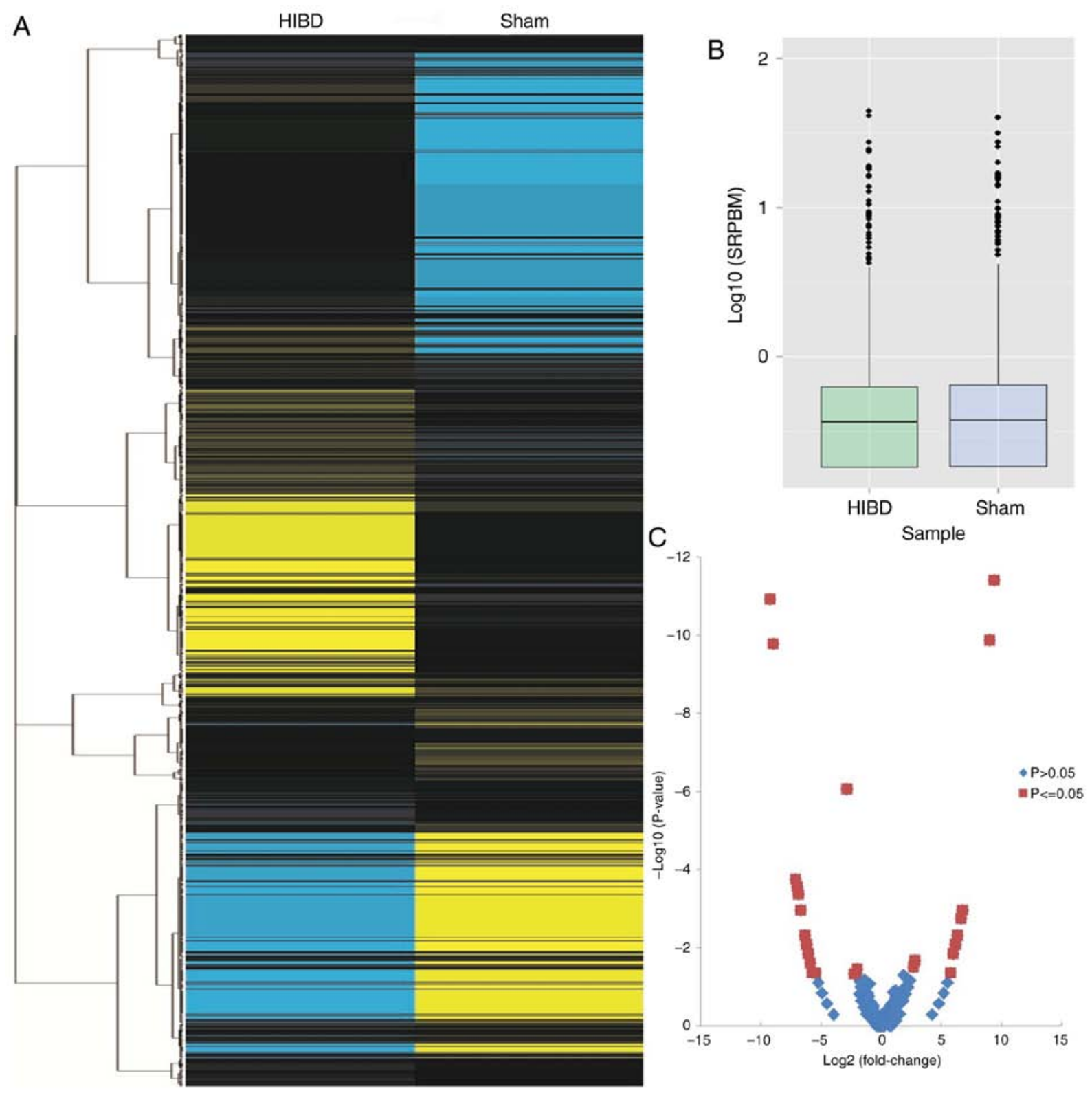

Figure 2. Altered expression profile of circRNAs in the hippocampus following HIBD. (A) Hierarchical clustering analysis of circRNAs that were differentially expressed between the sham and HIBD groups. (B) The distribution of the total expression of circRNAs between the sham and HIBD are nearly the same. (C) Volcano plots demonstrate the differentially expressed circRNAs with statistical significance (FC $>2.0$ and P $<0.05)$. circRNA, circular RNAs; HIBD, hypoxic-ischemic brain damage.

\section{Results}

CircRNAs expression profiles in neonatal rats with HIBD. RNA-Seq was used to investigate circRNAs expression profiles from the hippocampus of HIBD neonatal rats. Hierarchical clustering was performed to demonstrate the circRNAs expression pattern between the HIBD and control groups (Fig. 2A). As presented in the box plot, the distribution of the circRNAs expression profiles across all the samples are similar (Fig. 2B). Statistically significant alterations in circRNAs with between the sham and HIBD groups were identified using a volcano plot (Fig. 2C). Compared with the sham group, 66 circRNAs were demonstrated to be differentially expressed [fold change (FC) $>2.0$ and $\mathrm{P}<0.05$ ] following HIBD, including 20 upregulated and 46 downregulated circRNAs. The top 30 altered circRNAs are listed in Table II. The differentially expressed circRNAs following HIBD included $70 \%$ exonic and $28 \%$ intergenic in origin (Fig. 3A). The distributions of altered circRNAs revealed that the upregulated and downregulated circRNAs were transcribed from all chromosomes, with the exceptions of chr12, chr16 and chr20 (Fig. 3B). The downregulated circRNAs were transcribed from all chromosomes with the exceptions of chr12, chr13 chr16 and chr17 and the upregulated circRNAs were transcribed from most chromosomes (Fig. 3B).

CircRNAs gene symbols GO and pathway analysis. To determine the roles of circRNAs in physiological and pathological processes following HIBD, GO and KEGG pathway analyses were conducted for mRNAs transcribed from the parent genes of altered circRNAs. The results indicated that significant enriched and meaningful GO terms in biological process were 'positive regulation of guanosine monophosphatase 
Table II. The top 30 differential expressed circRNAs following hypoxic-ischemic brain damage.

\begin{tabular}{|c|c|c|c|c|c|}
\hline circRNA ID & $\operatorname{LogFC}$ & P-value & Chromosome & circRNA_type & Gene symbol \\
\hline chr1: 204959155|204961691 & 5.7672 & 0.04349 & chr1 & Intergenic & - \\
\hline chr13: 31092209|31209056 & 5.7672 & 0.04349 & $\operatorname{chr} 13$ & Exon & $\mathrm{Cdh} 7$ \\
\hline chr14: 82168512182184087 & 5.7672 & 0.04349 & chr14 & Intergenic & - \\
\hline chr17: 555549115571375 & 5.7672 & 0.04349 & chr17 & Exon & Agtpbp1 \\
\hline chrY:1142577|1153312 & 5.7672 & 0.04349 & $\operatorname{chrY}$ & Exon & Kdm6a \\
\hline chr1: 2008990661201028171 & 5.9858 & 0.01412 & $\operatorname{chr} 1$ & Intron & Ate1 \\
\hline chr1: 1565523401156969428 & 6.1756 & 0.00824 & $\operatorname{chr} 1$ & Exon & Dlg2 \\
\hline chr1: $235393349 \mid 235395737$ & 6.1756 & 0.00824 & chr1 & Exon & - \\
\hline chr1: 1958331891195883848 & 6.3433 & 0.00487 & $\operatorname{chr} 1$ & Intergenic & - \\
\hline chr2: 1205172041120520196 & 6.3433 & 0.00487 & $\operatorname{chr} 2$ & Intron & Dnajc19 \\
\hline chr19: 26268232|26303010 & 6.6295 & 0.00178 & chr19 & Intergenic & - \\
\hline chr2: 1779478481177973555 & 6.6295 & 0.00178 & $\operatorname{chr} 2$ & Exon & Rapgef2 \\
\hline chr1:195805819|195840151 & 6.7538 & 0.00109 & $\operatorname{chr} 1$ & Intergenic & - \\
\hline chr1: 1956581761195833285 & 9.0162 & 0.00000 & $\operatorname{chr} 1$ & Intergenic & - \\
\hline chr17:31482295|31493278 & 9.3864 & 0.00000 & $\operatorname{chr} 17$ & Intergenic & - \\
\hline chr11: 83225853183225993 & -9.2371 & 0.00000 & $\operatorname{chr} 11$ & Intron & Vps8 \\
\hline chr5: $90818736 \mid 90827570$ & -8.9711 & 0.00000 & chr5 & Intron & $\mathrm{Kdm} 4 \mathrm{c}$ \\
\hline chr3: $171790813 \mid 171790868$ & -7.1180 & 0.00018 & $\operatorname{chr} 3$ & Intergenic & - \\
\hline chr14: 108446849|108456923 & -6.9588 & 0.00027 & $\operatorname{chr} 14$ & Exon & Pus 10 \\
\hline chr3: 21894168I21898322 & -6.8721 & 0.00043 & $\operatorname{chr} 3$ & Exon & Strbp \\
\hline chr10: 59294314159298784 & -6.6812 & 0.00109 & $\operatorname{chr} 10$ & Exon & Ankfy1 \\
\hline chr11: $34065887 \mid 34070810$ & -6.6812 & 0.00109 & $\operatorname{chr} 11$ & Exon & Morc3 \\
\hline chr10:74336161174360926 & -6.3371 & 0.00487 & $\operatorname{chr} 10$ & Exon & Gdpd1 \\
\hline chr8: 79234306179249597 & -6.3371 & 0.00487 & chr8 & Exon & Rfx7 \\
\hline chr10: 13931236113935484 & -6.2014 & 0.00824 & $\operatorname{chr} 10$ & Exon & Pkd1 \\
\hline chr6: 29032002/29074704 & -6.2014 & 0.00824 & chr6 & Exon & Atad2b \\
\hline chr3: 112713524|112752649 & -6.0515 & 0.01412 & $\operatorname{chr} 3$ & Exon & Ttbk2 \\
\hline chr5: 1280830271128083901 & -6.0515 & 0.01412 & $\operatorname{chr} 5$ & Intergenic & - \\
\hline chr5: 148050939|148054556 & -6.0515 & 0.01412 & $\operatorname{chr} 5$ & Intergenic & - \\
\hline chr9: 53646697153648288 & -6.0515 & 0.01412 & $\operatorname{chr} 9$ & Exon & Mfsd6 \\
\hline
\end{tabular}
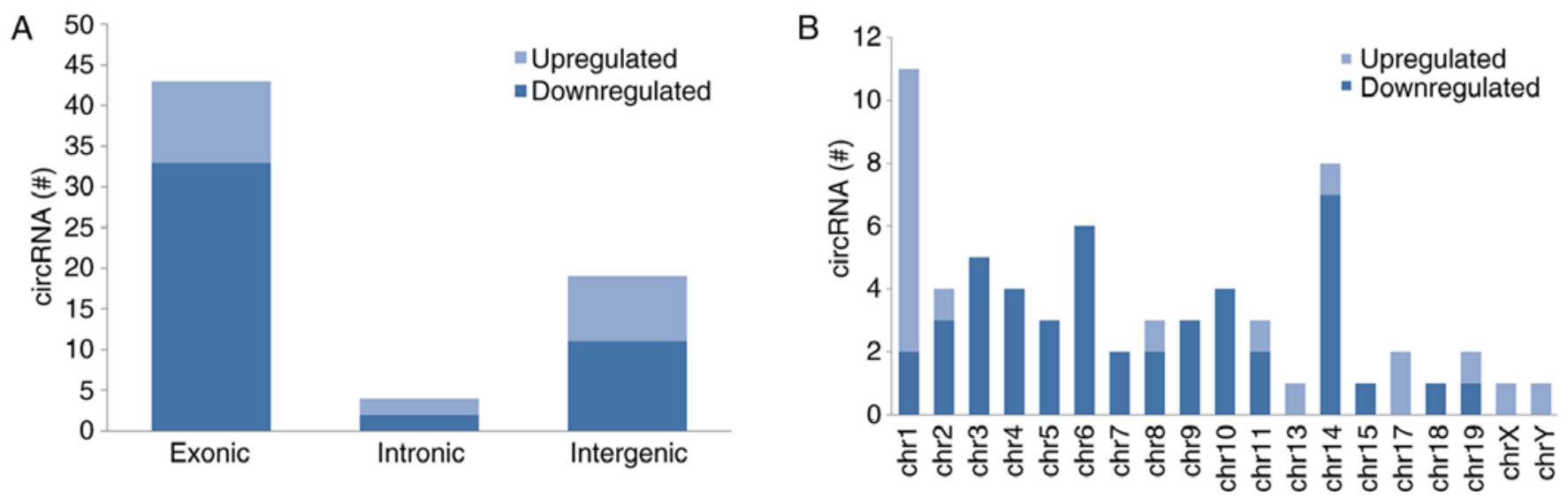

Figure 3. Features of altered circRNAs. (A) Most (98\%) of the circRNAs altered following HIBD are exonic and intergenic. (B) The distribution of altered circRNAs in rat chromosomes. circRNA, circular RNAs; HIBD, hypoxic-ischemic brain damage.

(GTPase) activity', 'intracellular signal transduction' and ' $\gamma$-aminobutyric acid (GABA) signaling pathway' (Fig. 4A). In terms of molecular function, the majority of the altered
circRNA associated mRNAs were identified to be 'protein binding', 'Rab GTPase binding' and 'adenosine triphosphate binding' (Fig. 4B). The cellular component (CC) included 

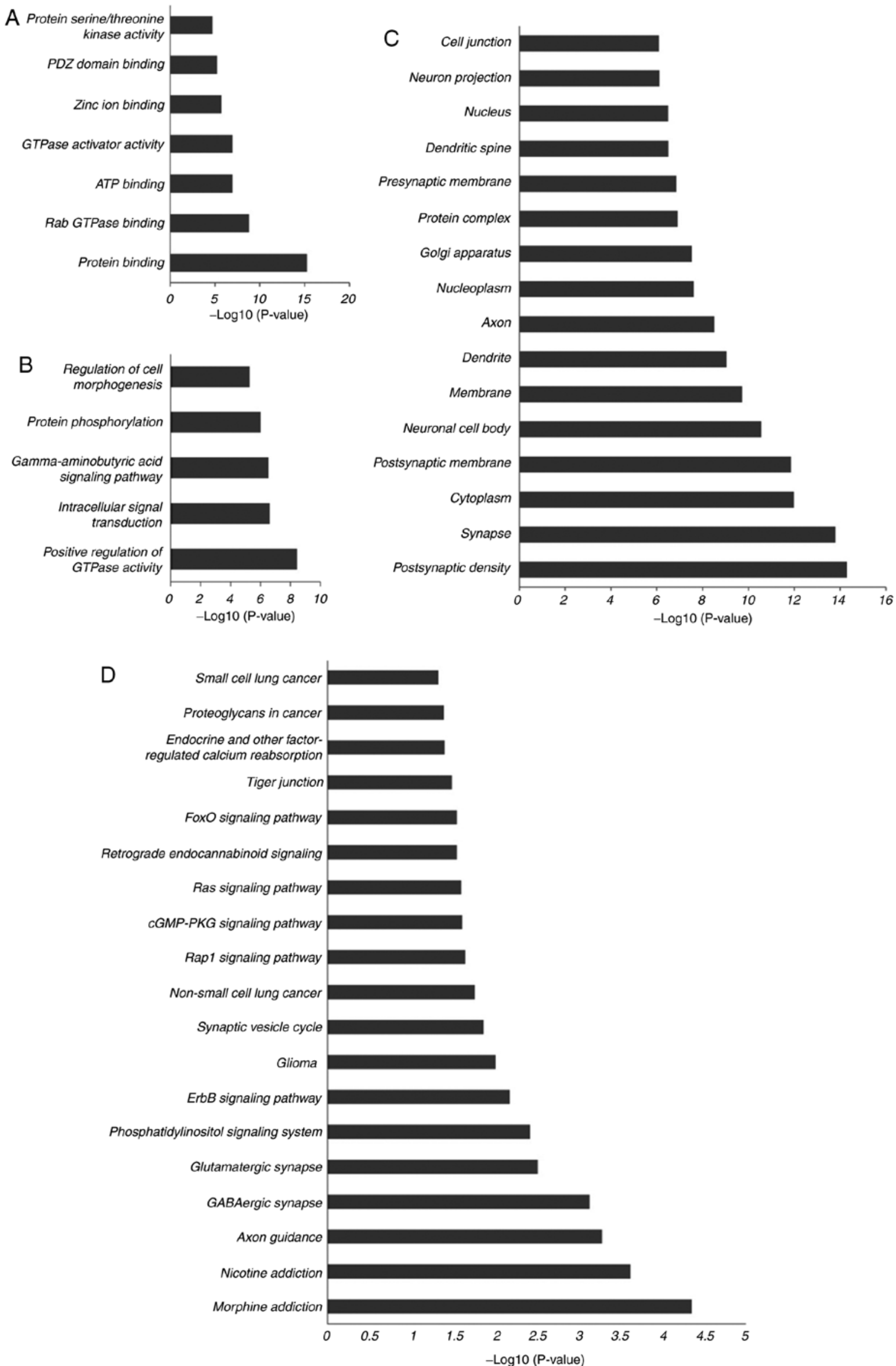

Figure 4. GO enrichment and pathway analysis of mRNAs originating from the host genes of altered circRNAs. (A) Major enriched and meaningful GO terms according to biological process. (B) Major enriched and meaningful GO terms according to molecular function. (C) Major enriched and meaningful GO terms according to cellular component. (D) The bar plot demonstrates the significantly enriched pathways. GO, gene ontology; circRNA, circular RNAs. 


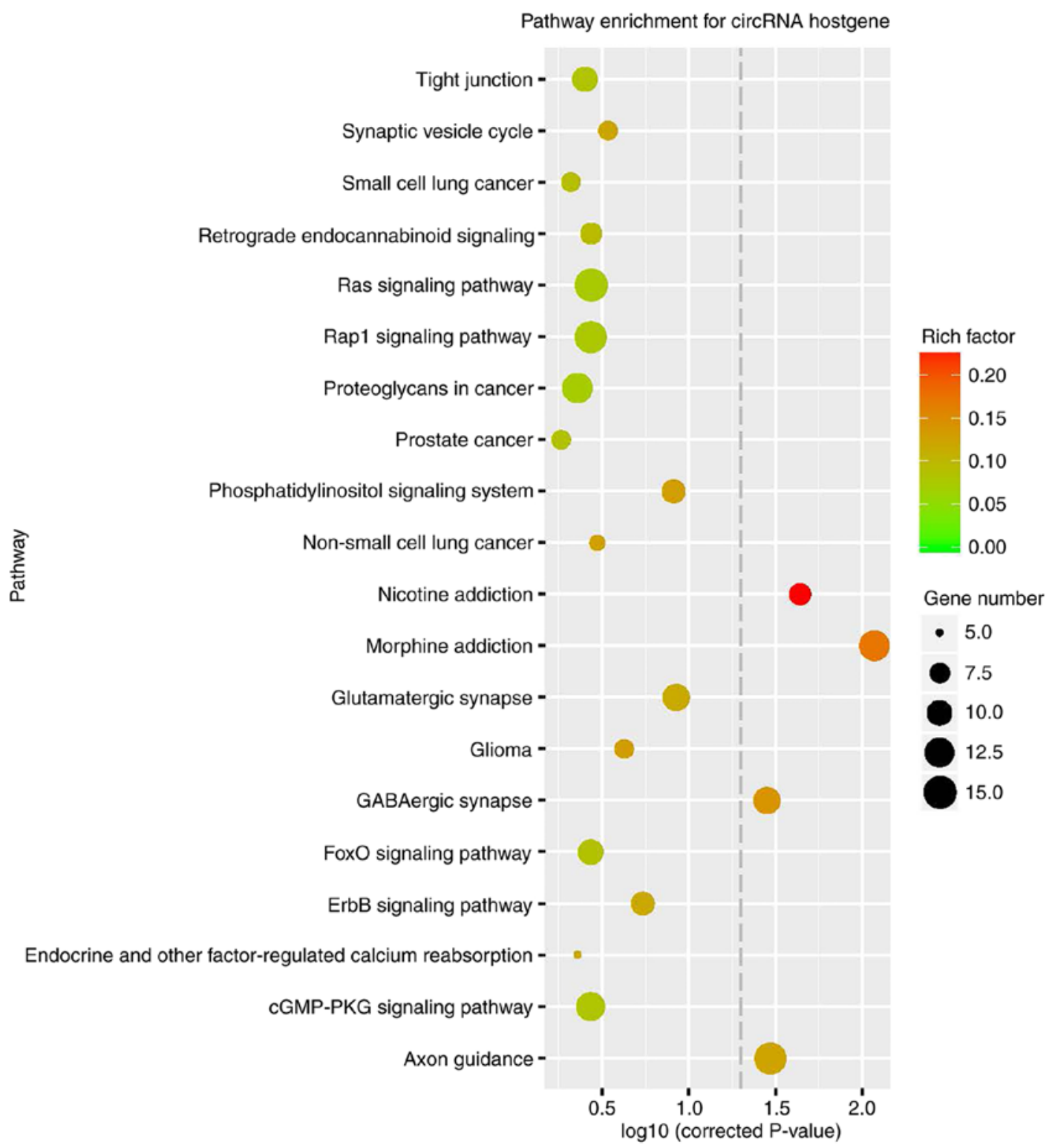

Figure 5. Kyoto Encyclopedia of Genes and Genomes pathway enrichment for the host gene of differentially expressed circRNAs. The majority of these host genes involved in axon guidance, cGMP-PKG signaling pathway, morphine addiction, Ras signaling pathway, Rap1 signaling pathway and GABAergic synapse. cGMP-PKG, cyclic guanine monophosphate-protein kinase G; GABA, $\gamma$-aminobutyric acid.

enriched CC terms, including 'postsynaptic density', 'synapse' and 'cytoplasm' (Fig. 4C). Gene-enriched KEGG pathway analysis suggested pathways that could be involved during the development of HIBD. The majority of these circRNAs were target genes involved in morphine addiction, nicotine addiction, axon guidance, GABAergic synapse and glutamatergic synapse (Figs. 4D and 5). Glutamatergic synapse and axon guidance have been reported to be involved in neuron injury and regeneration following brain injury in rats $(23,24)$.

$R T$ - $q P C R$ of circRNA. To verify the RNA-Seq data, 4 differentially expressed circRNAs were selected for qPCR and compared with the sham group, including circRNAs chr13: 31092209|31209056 and chr1: 2008990661201028171, which were upregulated, and chr11: 34065887134070810 and chr10: 13931236l13935484, which were downregulated significantly following HIBD ( $\mathrm{P}<0.01$; Fig. 6). The expression patterns of these circRNAs were consistent with the RNA-Seq data.
Annotation for circRNA/microRNA interaction. circRNAs serve a crucial role in sponging and regulating the expression of miRNA, their interaction with HIBD associated miRNAs suggests that circRNAs could serve an important role in pathophysiological processes of HIBD. Therefore, potential circRNA/microRNA interactions were investigated based on TargetScan and miRanda. The circRNA/miRNA interaction of the differentially expressed circRNAs is presented in Fig. 7. These results are in agreement with previously reported differential expression of miRNA in neonatal rats (25-29).

\section{Discussion}

To the best of the authors' knowledge, this is the first study to profile circRNA expression in a rat hippocampus following HIBD. Initially, 66 circRNAs were identified to be differentially expressed in the early stages following HIBD. Furthermore, significantly altered GO terms were identified, 
chr11:34065887|34070810

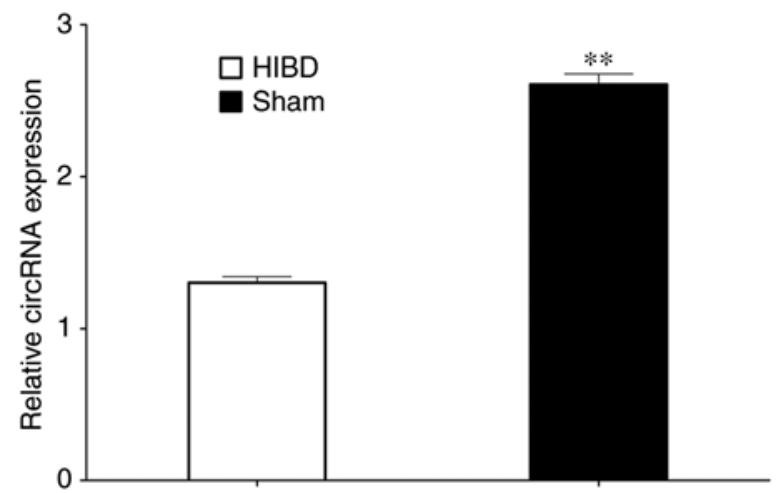

chr10:13931236|13935484

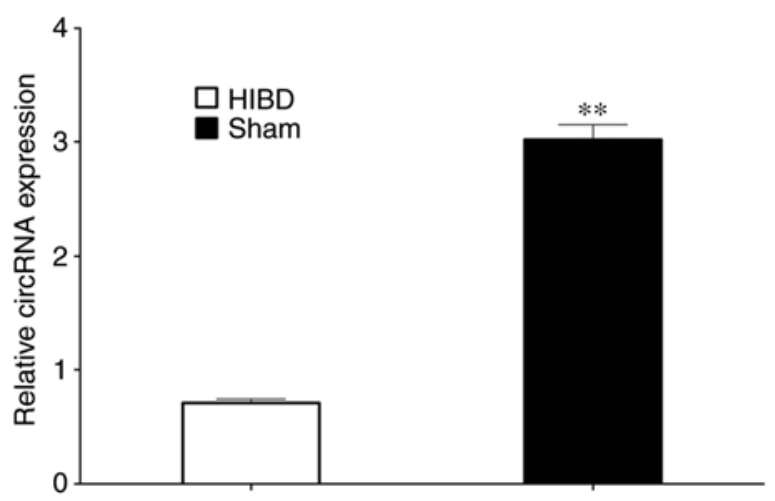

chr1:200899066|201028171

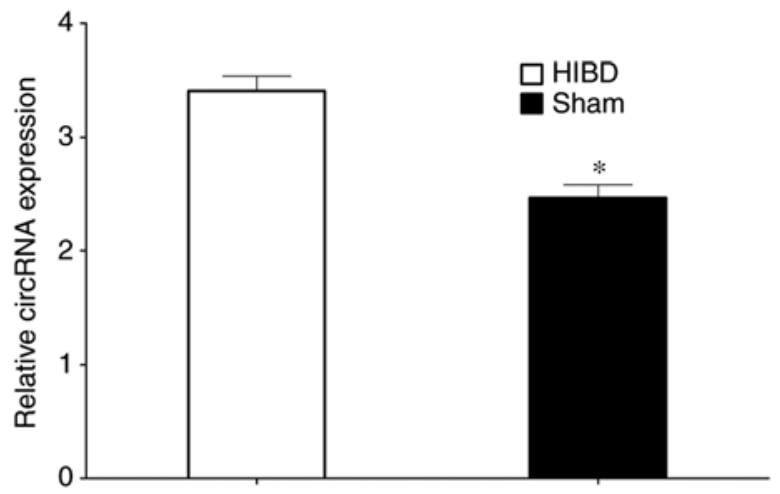

chr13:31092209|31209056

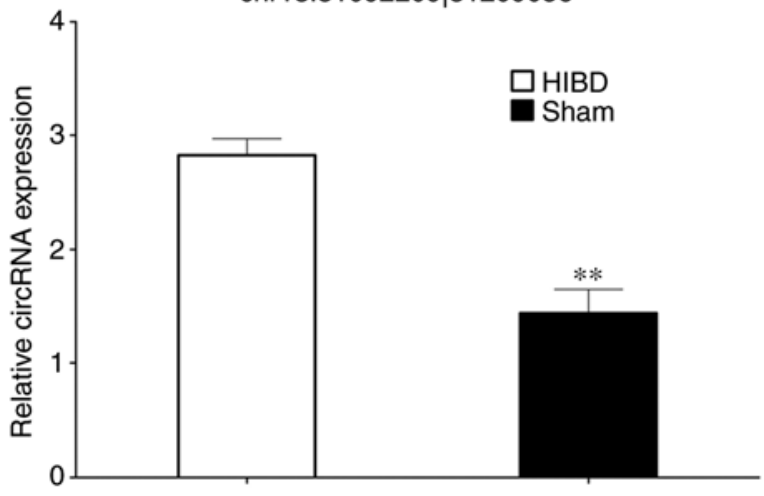

Figure 6. QPCR validation results of four selected circRNAs. A total of four circRNAs (chr1: 2008990661201028171, chr10: 13931236113935484, chr11: 34065887134070810 and chr13: 31092209131209056) were selected for validation by qPCR in eight samples. Data are presented as the mean \pm standard error of the mean ( $n=4$ per group). ${ }^{*} \mathrm{P}<0.05$ and ${ }^{* *} \mathrm{P}<0.01$ vs. the sham group (Student's $\mathrm{t}$ test). QPCR, quantitative polymerase chain reaction; circRNA, circular RNA; HIBD, hypoxia induced brain damage.
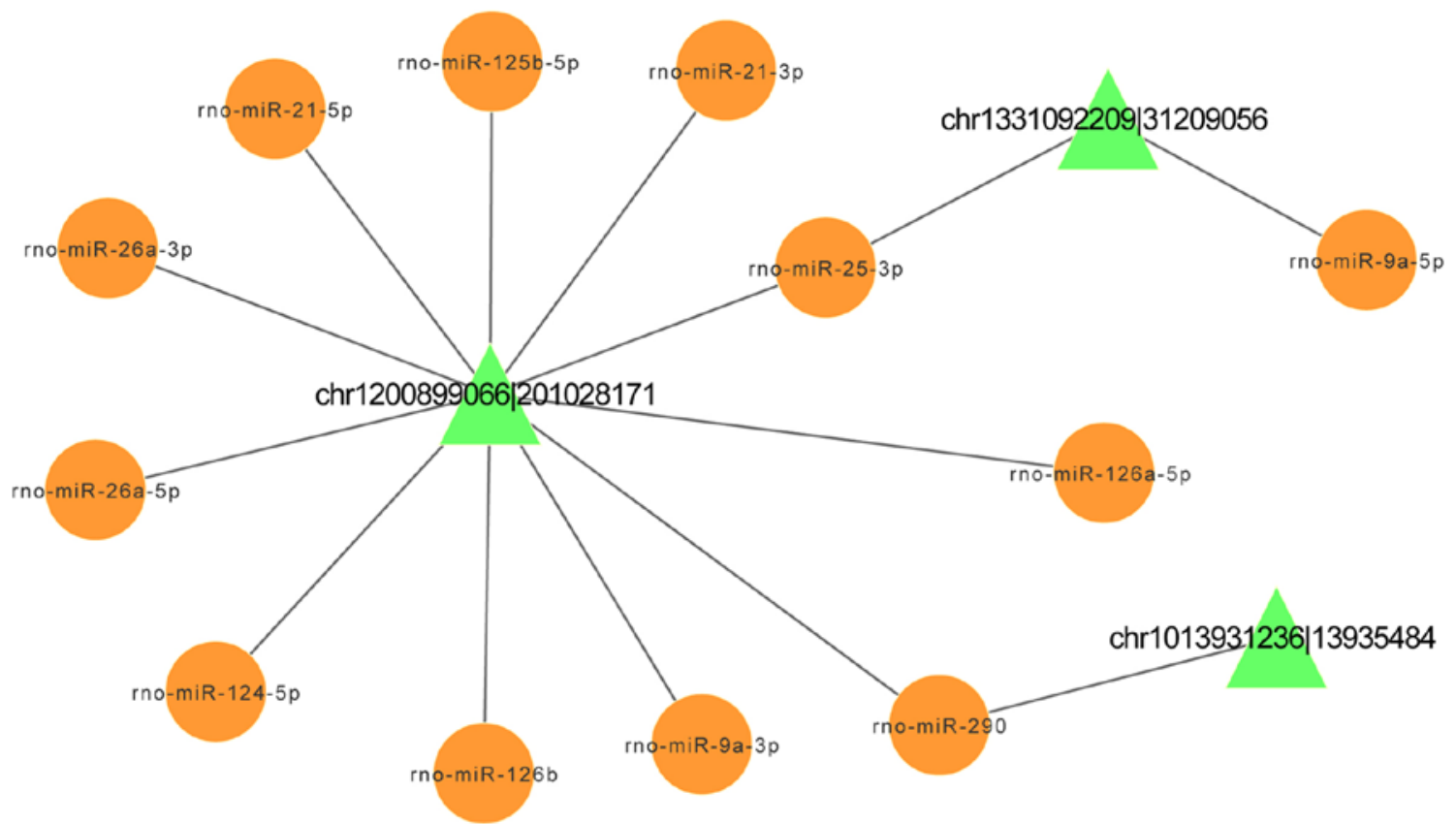

Figure 7. Annotation for circRNA/miRNA interaction. The orange nodes represent the differentially expressed miRNAs and the green nodes represent parts of the circRNAs identified to be differentially expressed in this study. Circ, circular; miRNA, microRNA.

mostly correlated pathways and predicted circRNA/miRNA interactions using bioinformatics analysis. A total of four
circRNAs were verified using RT-qPCR, which was consistent with the results from RNA-seq data. The potential roles 
of the altered circRNAs in the pathophysiological processes following HIBD suggest they could be potential biomarkers or novel therapeutic targets in the treatment of HIBD.

The reported evidence indicates that $>98 \%$ transcriptional output is not used in protein translation, but forms various classes of non-coding (nc)RNAs, including miRNAs, long non-coding RNAs and circRNAs (30). It is currently under debate whether ncRNAs have multiple functions, including controlling translation, transcription, epigenetics and RNA/protein scaffolding. In general, ncRNAs are considered to have novel roles in genomic regulation and dysregulation of ncRNAs is associated with the onset of disease and secondary neural damage following central nervous system (CNS) injury. Notably, several pathophysiological mechanisms, including inflammation, blood-brain damage, apoptosis, autophagy, oxidative stress and endoplasmic reticulum stress are closely associated with the expression of ncRNAs, it is suggested that the brain damage may be corrected by determining the level of dysregulated ncRNAs (31-34). Therefore, reversing the altered ncRNAs could be a potential therapeutic strategy for HIBD.

miR-126 was significantly upregulated following HIBD, which was in agreement with our previously report; miR-126 expression levels were associated with retinopathy of premature neonatal rats induced by acidosis, in which mRNA and protein expression levels of the vascular endothelial growth factor were notably upregulated $(35,36)$. Similar results were identified for miR-9a, which was reported to be significantly increased in the plasma of hyperoxia-induced neonatal rats and involved in 'in utero embryonic development', 'neurotrophin signaling pathway', 'B cell receptor signaling pathway' and 'mitogen activated protein kinase signaling pathway'. On the contrary, miR-21a and miR-25a were downregulated in the injured cortex following hypoxic-ischemic brain damage and contribute to neuronal death with increased expression levels of the proapoptotic B-cell lymphoma 2 family members-Noxa and Bax. In contrast, increasing the expression levels of these miRNAs significantly mitigates neural damage $(37,38)$.

Overall, the results from this study suggest potential in the treatment of HIBD using targeted miRNAs and lncRNAs. However, the role of circRNAs, a new star of ncRNAs, in the pathophysiological process of HIBD is yet to be reported. The expression profiles of circRNA in this study were altered following HIBD. As previous studies report, circRNAs can regulate the transcription of their host genes $(39,40)$ and dysregulation could influence numerous molecular events essential to the process of brain damage following HIBD. Parent genes that generate HIBD-altered circRNAs primarily participated in the positive regulation of guanosine triphosphatase activity, intracellular signal transduction and GABA signaling pathway, which are indispensable for neural regeneration. Synapse, neuron part and neuron projection are essential for neuron-neuron communication, producing movement, feeling and memory. Altered circRNA-associated mRNAs were primarily located in the synapse, and the neuron to neuron projection, which may be correlated to post-HIBD pathophysiology. However, further investigation is required to determine the regulation of circRNAs in HIBD.

CircRNAs are reported to act as miRNA sponges and suppress miRNA activity, resulting in upregulation of miRNA targets $(5,8,41)$. miRNAs serves key roles in normal CNS development and function $(42,43)$ and certain circRNAs could be involved in HIBD through circRNA/miRNA interactions. The dysregulated circRNAs following HIBD was demonstrated to include several miRNA binding sites using miRNA target prediction software. The correlation of miRNAs with HIBD suggest that circRNAs could serve important roles in HIBD. For example, chr1: 200899066/201028171 was predicted to bind miR-126a, miR-9a and miR-26a and miR-9a was identified to be associated with hypoxia-induced neuronal apoptosis (26,44-46).

In conclusion, the results in this study indicated that circRNAs were significantly altered in the hippocampus following HIBD compared with the sham control. Using bioinformatics analysis and circRNA/miRNA interaction prediction, circRNAs could be involved in brain damage and also neural regeneration following HIBD. Therefore, these findings suggest a potential treatment of HIBD through the modulation of circRNAs. Deciphering the biological functions of the circRNAs identified in this study requires further investigation.

\section{Acknowledgements}

Not applicable.

\section{Funding}

The present study was financially supported by the Project of Clinical Advanced Techniques, the Primary Research and Development Plan of Jiangsu Province (grant no. BE2017719), the Pediatric Medical Innovation Team of Jiangsu Province (grant no. CXTDA2017022), the Project of National Youth Fund (grant no. 81601355) and the Project of Postdoctoral Fund of Jiangsu Province (grant no. 1701162C).

\section{Availability of data and materials}

The datasets used in this study are available from the corresponding author on reasonable request.

\section{Authors' contributions}

LJ, RZ and ZX contributed to the concept and design of the present study, prepared the manuscript and conducted the experiments and data analysis. HL and RZ performed animal treatments. RZ contributed to the acquisition of data. LJ and ZF conducted bioinformatic analysis. ZX also provided guidances. All the authors read and approved the final version of the manuscript.

\section{Ethics approval and consent to participate}

All animal procedures were approved by the Zhongda Hospital Committee on Animal Research and all the experiments were carried out in accordance with the approved guidelines.

\section{Patient consent for publication}

Not applicable. 


\section{Competing interests}

The authors declare that there are no known competing interests regarding the publication of this study.

\section{References}

1. Wachtel EV and Hendricks-Munoz KD: Current management of the infant who presents with neonatal encephalopathy. Curr Probl Pediatr Adolesc Health Care 41: 132-153, 2011.

2. Ashwal-Fluss R, Meyer M, Pamudurti NR, Ivanov A, Bartok O, Hanan M, Evantal N, Memczak S, Rajewsky N and Kadener S: circRNA biogenesis competes with pre-mRNA splicing. Mol Cell 56: 55-66, 2014.

3. Qu S, Yang X, Li X, Wang J, Gao Y, Shang R, Sun W, Dou K and Li H: Circular RNA: A new star of noncoding RNAs. Cancer Lett 365: 141-148, 2015

4. Rybak-Wolf A, Stottmeister C, Glažar P, Jens M, Pino N, Giusti S, Hanan M, Behm M, Bartok O, Ashwal-Fluss R, et al: Circular RNAs in the mammalian brain are highly abundant, conserved, and dynamically expressed. Mol Cell 58: 870-885, 2015.

5. Lasda E and Parker R: Circular RNAs: Diversity of form and function. RNA 20: 1829-1842, 2014.

6. Li X, Yang L and Chen LL: The biogenesis, functions, and challenges of circular RNAs. Mol Cell 71: 428-442, 2018.

7. Zheng Q, Bao C, Guo W, Li S, Chen J, Chen B, Luo Y, Lyu D, $\mathrm{Li}$ Y, Shi G, et al: Circular RNA profiling reveals an abundant circHIPK3 that regulates cell growth by sponging multiple miRNAs. Nat Commun 7: 11215, 2016.

8. Hansen TB, Jensen TI, Clausen BH, Bramsen JB, Finsen B, Damgaard CK and Kjems J: Natural RNA circles function as efficient microRNA sponges. Nature 495: 384-388, 2013.

9. Memczak S, Jens M, Elefsinioti A, Torti F, Krueger J, Rybak A, Maier L, Mackowiak SD, Gregersen LH, Munschauer M, et al: Circular RNAs are a large class of animal RNAs with regulatory potency. Nature 495: 333-338, 2013

10. Meng S, Zhou H, Feng Z, Xu Z, Tang Y,Li P and Wu M: CircRNA: Functions and properties of a novel potential biomarker for cancer. Mol Cancer 16: 94, 2017.

11. Hansen TB, Kjems J and Damgaard CK: Circular RNA and miR-7 in cancer. Cancer Res 73: 5609-5612, 2013.

12. Mehta SL, Pandi G and Vemuganti R: Circular RNA expression profiles alter significantly in mouse brain after transient focal ischemia. Stroke 48: 2541-2548, 2017.

13. Liu C, Zhang C, Yang J, Geng X, Du H, Ji X and Zhao H: Screening circular RNA expression patterns following focal cerebral ischemia in mice. Oncotarget 8: 86535-86547, 2017.

14. Dharap A, Nakka VP and Vemuganti R: Effect of focal ischemia on long noncoding RNAs. Stroke 43: 2800-2802, 2012.

15. Wang Z, Gerstein M and Snyder M: RNA-Seq: A revolutionary tool for transcriptomics. Nat Rev Genet 10: 57-63, 2009.

16. Zhao F, Qu Y, Xiong T, Duan Z, Ye Q and Mu D: The neuroprotective role of TERT via an antiapoptotic mechanism in neonatal rats after hypoxia-ischemia brain injury. Neurosci Lett 515: 39-43, 2012

17. Trapnell C, Roberts A, Goff L, Pertea G, Kim D, Kelley DR, Pimentel H, Salzberg SL, Rinn JL and Pachter L: Differential gene and transcript expression analysis of RNA-seq experiments with TopHat and Cufflinks. Nat Protoc 7: 562-578, 2012.

18. Zhu L, Ni W, Liu S, Cai B, Xing H and Wang S: Transcriptomics analysis of apple leaves in response to alternaria alternata apple pathotype infection. Front Plant Sci 8: 22, 2017.

19. Wang Z, Ruan B, Jin Y, Zhang Y, Li J, Zhu L, Xu W, Feng L, Jin H and Wang X: Identification of KLK10 as a therapeutic target to reverse trastuzumab resistance in breast cancer. Oncotarget 7 : 79494-79502, 2016

20. Harrow J, Frankish A, Gonzalez JM, Tapanari E, Diekhans M, Kokocinski F, Aken BL, Barrell D, Zadissa A, Searle S, et al: GENCODE: The reference human genome annotation for The ENCODE project. Genome Res 22: 1760-1774, 2012.

21. Quinlan AR and Hall IM: BEDTools: A flexible suite of utilities for comparing genomic features. Bioinformatics 26: 841-842, 2010.

22. Livak KJ and Schmittgen TD: Analysis of relative gene expression data using real-time quantitative PCR and the 2(-Delta Delta C(T)) method. Methods 25: 402-408, 2001.

23. Hollis ER II: Axon guidance molecules and neural circuit remodeling after spinal cord injury. Neurotherapeutics 13: 360-369, 2016

24. Park K and Biederer T: Neuronal adhesion and synapse organization in recovery after brain injury. Future Neurol 8: 555-567, 2013
25. Dang YX, Shi KN and Wang XM: Early changes in glutamate metabolism and perfusion in basal ganglia following hypoxia-ischemia in neonatal piglets: A multi-sequence $3.0 \mathrm{~T}$ MR study. Front Physiol 8: 237, 2017.

26. Cai Q, Wang T, Yang WJ and Fen X: Protective mechanisms of microRNA-27a against oxygen-glucose deprivation-induced injuries in hippocampal neurons. Neural Regen Res 11: 1285-1292, 2016

27. Yin X, Li L, Zhang X, Yang Y, Chai Y, Han X and Feng Z: Development of neural stem cells at different sites of fetus brain of different gestational age. Int J Clin Exp Pathol 6: 2757-2764, 2013.

28. Filipowicz W, Bhattacharyya SN and Sonenberg N: Mechanisms of post-transcriptional regulation by microRNAs: Are the answers in sight? Nat Rev Genet 9: 102-114, 2008.

29. Zhao R, Qian L and Jiang L: Identification of retinopathy of prematurity related miRNAs in hyperoxia-induced neonatal rats by deep sequencing. Int J Mol Sci 16: 840-856, 2014.

30. Chandran R, Mehta SL and Vemuganti R: Non-coding RNAs and neuroprotection after acute CNS injuries. Neurochem Int 111: 12-22, 2017

31. Gaudet AD, Fonken LK, Watkins LR, Nelson RJ and Popovich PG: MicroRNAs: Roles in regulating neuroinflammation. Neuroscientist 24: 221-245, 2018

32. Ge X, Han Z, Chen F, Wang H, Zhang B, Jiang R, Lei P and Zhang J: MiR-21 alleviates secondary blood-brain barrier damage after traumatic brain injury in rats. Brain Res 1603: 150-157, 2015.

33. Han Z, Chen F, Ge X, Tan J, Lei P and Zhang J: miR-21 alleviated apoptosis of cortical neurons through promoting PTEN-Akt signaling pathway in vitro after experimental traumatic brain injury. Brain Res 1582: 12-20, 2014.

34. Redell JB, Zhao J and Dash PK: Altered expression of miRNA-21 and its targets in the hippocampus after traumatic brain injury. J Neurosci Res 89: 212-221, 2011.

35. Zhao R, Hou W, Zhang Z, Qian L and Jiang L: Differential expression of Mir-1 26 and vascular endothelial growth factor in retinal cells of metabolic acidosis-induced neonatal rats. J Nanosci Nanotechnol 15: 2088-2093, 2015.

36. Zhao RB, Qian LJ and Jiang L: miRNA-dependent cross-talk between VEGF and Ang-2 in hypoxia-induced microvascular dysfunction. Biochem Biophys Res Commun 452: 428-435, 2014.

37. Sabirzhanov B, Zhao Z, Stoica BA, Loane DJ, Wu J, Borroto C, Dorsey SG and Faden AI: Downregulation of miR-23a and miR-27a following experimental traumatic brain injury induces neuronal cell death through activation of proapoptotic Bcl-2 proteins. J Neurosci 34: 10055-10071, 2014.

38. Zhong J, Jiang L, Cheng C, Huang Z, Zhang H, Liu H, He J, Cao F, Peng J, Jiang Y and Sun X: Altered expression of long non-coding RNA and mRNA in mouse cortex after traumatic brain injury. Brain Res 1646: 589-600, 2016.

39. Zhang Y, Zhang XO, Chen T, Xiang JF, Yin QF, Xing YH, Zhu S, Yang $L$ and Chen LL: Circular intronic long noncoding RNAs. Mol Cell 51: 792-806, 2013.

40. Li Z, Huang C, Bao C, Chen L, Lin M, Wang X, Zhong G, Yu B, $\mathrm{Hu}$ W, Dai L, et al: Exon-intron circular RNAs regulate transcription in the nucleus. Nat Struct Mol Biol 22: 256-264, 2015.

41. Ebbesen KK, Kjems J and Hansen TB: Circular RNAs: Identification, biogenesis and function. Biochim Biophys Acta 1859: 163-168, 2016.

42. Bhalala OG, Srikanth M and Kessler JA: The emerging roles of microRNAs in CNS injuries. Nat Rev Neurol 9: 328-339, 2013.

43. Liu NK and Xu XM: MicroRNA in central nervous system trauma and degenerative disorders. Physiol Genomics 43: 571-580, 2011.

44. Chen Q, Xu J, Li L, Li H, Mao S, Zhang F, Zen K, Zhang CY and Zhang Q: MicroRNA-23a/b and microRNA-27a/b suppress Apaf-1 protein and alleviate hypoxia-induced neuronal apoptosis. Cell Death Dis 5: e1132, 2014.

45. Chen Q, Zhang F, Wang Y, Liu Z, Sun A, Zen K, Zhang CY and Zhang Q: The transcription factor c-Myc suppresses MiR-23b and MiR-27b transcription during fetal distress and increases the sensitivity of neurons to hypoxia-induced apoptosis. PLoS One 10: e0120217, 2015.

46. Shin JH, Park YM, Kim DH, Moon GJ, Bang OY, Ohn T and Kim HH: Ischemic brain extract increases SDF-1 expression in astrocytes through the CXCR $2 / \mathrm{miR}-223 / \mathrm{miR}-27 \mathrm{~b}$ pathway. Biochim Biophys Acta 1839: 826-836, 2014.

This work is licensed under a Creative Commons Attribution-NonCommercial-NoDerivatives 4.0 International (CC BY-NC-ND 4.0) License. 